\title{
Compostos fitogênicos e substâncias bioativas para coelhos: benefícios sobre a reprodução, bem-estar e qualidade da carne
}

\author{
Phytogenic compounds and bioactive substances for rabbits: benefits on reproduction, well-being \\ and meat quality
}

Compuestos fitogénicos y sustancias bioactivas para conejos: beneficios sobre la reproducción, el bienestar y la calidad de la carne

Vítor Magalhães de Mendonça Cunha Miranda ORCID: https://orcid.org/0000-0001-7473-4398 Universidade Estadual de Maringá, Brasil E-mail: vitor.zootec01@gmail.com

Letícia Aline Lima da Silva ORCID: https://orcid.org/0000-0002-4758-9890 Universidade Estadual de Maringá, Brasil E-mail: leticiaalinezoo@gmail.com Sílvio Mayke Leite ORCID: https://orcid.org/0000-0002-1803-2698 Universidade Estadual de Maringá, Brasil E-mail: silviomaykeleite@gmail.com Beatriz Lazaretti Ribeiro ORCID: https://orcid.org/0000-0002-0026-0131 Universidade Estadual de Maringá, Brasil E-mail: Lazarettibeatriz1@gmail.com

Polyana Roeles Batista ORCID: https://orcid.org/0000-0003-3703-803X Universidade Estadual de Maringá, Brasil E-mail: polyanab23@gmail.com

Edson Massayuki Tokusumi Teotonho da Silva ORCID: https://orcid.org/0000-0002-5352-4989 Universidade Estadual de Maringá, Brasil E-mail: massatks@gmail.com Leandro Dalcin Castilha ORCID: https://orcid.org/0000-0003-4799-2839 Universidade Estadual de Maringá, Brasil E-mail: ldcastilha@uem.br

\begin{abstract}
Resumo
A utilização de aditivos fitogênicos na alimentação animal surgiu com a necessidade de substituição dos aditivos tradicionais e os promotores de crescimento, que foram proibidos para utilização pela União Europeia há alguns anos e no Brasil recentemente. Desde então, vários trabalhos foram realizados para diversas espécies da produção animal, de modo que atualmente os aditivos fitogênicos mais utilizados são os óleos essenciais, extratos, ervas, especiarias e frações vegetais que incluem cascas, folhas, raízes, sementes e outros. Estes aditivos são compostos por várias substâncias bioativas de natureza química variada, exercendo funções positivas sobre o organismo dos animais. Na cunicultura, são utilizadas principalmente para melhorar o funcionamento do sistema digestório, reduzir desafio sanitário e promover maior digestibilidade de nutrientes. Além de melhorar a nutrição, o consumo, estimular a digestão e o desempenho, o uso de substâncias bioativas reduz a incidência de doenças, melhora os parâmetros reprodutivos, atuam como antifúngicos, anti-helmínticos, antivirais, antimicrobianos, anticoccidianos e antioxidantes. Devido a esses benefícios, o objetivo desta revisão é abordar os diferentes tipos de compostos fitogênicos e substâncias bioativas utilizadas na produção de coelhos dando enfoque na reprodução, bem-estar e qualidade da carne. Palavras-chave: Aditivos naturais; Antioxidantes; Cunicultura.
\end{abstract}

\section{Abstract}

The use of phytogenic additives in animal feed emerged with the need to replace traditional additives and growth promoters, which were banned for use by the European Union a few years ago and in Brazil recently. Since then, several works have been carried out for several species of animal production, so that currently the most used phytogenic additives are essential oils, extracts, herbs, spices and vegetable fractions that include bark, leaves, roots, 
seeds and others. These additives are composed of several bioactive substances of varied chemical nature, exerting positive functions on the animals' organism. In rabbit farming, they are mainly used to improve the functioning of the digestive system, reduce sanitary challenge and promote greater nutrient digestibility. In addition to improving nutrition, consumption, stimulating digestion and performance, the use of bioactive substances reduces the incidence of diseases, improves reproductive parameters, acts as antifungals, anthelmintics, antivirals, antimicrobials, anticoccidials and antioxidants. Due to these benefits, the current review addresses different types of phytogenic compounds and bioactive substances used in rabbit production, focusing on reproduction, welfare and meat quality.

Keywords: Antioxidants; Natural Additives; Rabbit Farming.

\section{Resumen}

El uso de aditivos fitogénicos en la alimentación animal surgió con la necesidad de reemplazar los aditivos tradicionales y los promotores del crecimiento, cuyo uso fue prohibido por la Unión Europea hace unos años y recientemente en Brasil. Desde entonces, se han realizado varios trabajos para diversas especies de producción animal, por lo que actualmente los aditivos fitogénicos más utilizados son los aceites esenciales, extractos, hierbas, especias y fracciones vegetales que incluyen corteza, hojas, raíces, semillas y otros. Estos aditivos están compuestos por varias sustancias bioactivas de diversa naturaleza química, que ejercen funciones positivas sobre el organismo de los animales. En la cría de conejos, se utilizan principalmente para mejorar el funcionamiento del sistema digestivo, reducir el desafío sanitario y promover una mayor digestibilidad de los nutrientes. Además de mejorar la nutrición, el consumo, estimular la digestión y el rendimiento, el uso de sustancias bioactivas reduce la incidencia de enfermedades, mejora los parámetros reproductivos, actúa como antifúngicos, antihelmínticos, antivirales, antimicrobianos, anticoccidiales y antioxidantes. Debido a estos beneficios, el objetivo de esta revisión es abordar los diferentes tipos de compuestos fitogénicos y sustancias bioactivas que se utilizan en la producción de conejos, centrándose en la reproducción, el bienestar y la calidad de la carne.

Palabras clave: Aditivos naturales; Antioxidantes; Cría de conejos.

\section{Introdução}

A cunicultura é um ramo que possui diversos propósitos como a produção de carne, pele, pesquisas, pets entre outros. Tornando-o assim um animal com potencial para grandes e pequenos produtores. Acrescido a isso tem a facilidade de manejo destes animais, alta prolificidade, curtos períodos de reprodução (Cruz et al., 2020). Entretanto, a carne de coelho ainda é pouco consumida no Brasil, isso pode ser relacionado com a falta da oferta da carne, alto preço e falta de organização do setor produtivo.

$\mathrm{Na}$ alimentação dos coelhos é comum a utilização de aditivos antioxidantes como BHT e BHA, no combate a oxidação dos alimentos, além dos anticoccidianos. Os aditivos na alimentação animal têm sido utilizados com o intuito de serem melhoradores da qualidade do alimento, dos produtos de origem animal ou atuarem como promotores de crescimento.

Devido às proibições na exportação da carne com o uso de aditivos sintéticos e promotores de crescimento, tem-se buscado substituir tais aditivos por produtos naturais, que não deixam resíduos nos produtos. Esses produtos naturais são os compostos fitogênicos, que são ofertados na alimentação animal de formas diferentes para qualquer espécie. Segundo Karásková, Suchý e Straková. (2015), estes compostos têm obtido tamanha visibilidade devido ao fato que além de promoverem saúde aos animais consumidores, ainda atuam desempenhando o papel de todos os tipos de aditivos mencionados na normativa 13 do Mapa (BRASIL, 2004), que são: Tecnológicas, Sensoriais, Nutricionais e Zootécnicas.

Os compostos fitogênicos estão presentes em uma gama de plantas e extrativos, muitos dos quais têm sido utilizados na alimentação humana e animal como antioxidantes, medicamentos ou como componentes do sabor. Dentro destes compostos estão as propriedades bioativas dos alimentos, como por exemplo, os flavonoides e compostos fenólicos (Kutlu \& Erdooan, 2010).

Estas substâncias são comumente encontradas em ervas, especiarias, óleos essenciais, assim como extrativos vegetais em grande quantidade, e sua utilização nos alimentos ou rações permite que seja inserida na forma sólida, moída, crua ou concentrada (Gadde et al., 2017). Entretanto sua disponibilidade no alimento estará associada à fração da planta e principalmente ao processo de extração de tais princípios ativos (Yitbarek, 2015). 
Esta revisão de literatura foi elaborada com o objetivo de discorrer acerca das propriedades dos compostos fitogênicos e substâncias bioativas na alimentação de coelhos, dando enfoque na reprodução, bem-estar e qualidade da carne.

\section{Metodologia}

Para esta revisão foi realizada uma pesquisa do tipo qualitativa segundo Pereira et al. (2018), os artigos selecionados foram extraidos de diferentes bases de dados, sendo: Elsevier, Pubmed, Scielo, periódicos Capes, Science Direct, Google Scholar e Scopus. Com recorte temporal de 1998 a 2021.

Os artigos foram classificados utilizando como tema principal os compostos fitogênicos e substancias bioativas para coelhos, com o enfoque na reprodução, bem-estar e qualidade da carne. Como palavras chaves foram pesquisadas: compostos bioativos para coelhos, substancias bioativas para coelhos, e para cada um desses foram inseridos as palavras reprodução, bemestar e qualidade de carne. Como critério de seleção para idioma, foram utilizados os artigos em português e inglês, que abordassem a relevância do tema em questão, além da utilização de livros e leis para o embasamento dos conceitos. No processo de pré-seleção foram coletados 105 artigos, entretanto alguns deles apresentavam objetivos diferentes, então como critério de exclusão, foram retirados 25 destes artigos. Portanto, foram utilizados para a realização desta revisão 77 artigos, 1 lei e 2 livros cujas informações eram pertinentes sobre o assunto abordado neste material.

\section{Compostos Fitogênicos}

Os aditivos fitogênicos são conhecidos na literatura como produtos nutracêuticos ou fitobióticos originados de metabólitos das plantas que são adicionados na alimentação dos animais (Koiyama et al., 2012). Eles são classificados como: ervas (utilização de flores e plantas não lenhosas), óleo resinas (extratos com base em um solvente não aquoso), óleos essenciais (extração de hidro destilados de compostos vegetais voláteis) e botânicas (plantas inteiras ou frações como raízes, folhas e cascas).

De acordo com Madhupriya et al. (2018), estes aditivos podem ser fornecidos de acordo com a forma de utilização, sendo na forma líquida (extratos e óleos essenciais) ou mesmo sólida (seca, triturada ou moída). Além disso, sua composição é dependente de uma série de fatores, como por exemplo: condições climáticas, stress exógeno, posição geográfica, forma de colheita, armazenamento e principalmente a forma de extração do material desejado.

Vários autores na literatura têm realizados trabalhos com tais compostos em animais monogástricos e entre as principais melhorias nos dados zootécnicos encontrados estão: melhor digestibilidade dos alimentos (Maenner, Vahjen \& Simon., 2011), maior comprimento das bordas intestinais (Cardoso et al., 2012), maiores atividades antioxidantes (Zhang et al., 2013), efeitos hipocolesterolêmicos (Cho, Kim \& Kim et al., 2014), melhoria nos rendimentos de carcaça e cortes comerciais (Khattak et al., 2014) além de atuar no controle de microrganismos patogênicos presentes no sistema digestivo (Cho, Kim \& Kim et al., 2014).

\section{1 Óleos Essenciais}

Os óleos são os mais conhecidos entre os compostos fitogênicos na literatura, possuindo diferentes métodos de extração (fermentação, destilação ou à vapor) e diferentes frações das plantas como: flores, sementes, galhos, folhas, frutas, raízes entre outras, onde a sua extração e a fração modificam a sua função (Gopi, 2014).

Apesar de serem bastante utilizados apresentam algumas limitações por serem voláteis. Seus principais efeitos são as atividades antioxidantes, antimicrobianos, melhorando assim a digestão e absorção dos nutrientes, além de atuar como um alimento natural em substituição aos aditivos promotores de crescimento (Jayasena \& Jo, 2013; Valero et al., 2014). 
Segundo Mueller et al. (2012), os óleos essenciais de orégano, alecrim e tomilho são capazes de estimular uma maior regulação de um gene ARE (antioxidante-response elemento) capaz de aumentar a produção de enzimas oxidantes no organismo. Estes óleos possuem uma forma de atuar diferente dependendo da posição do grupamento hidroxila, pois é este radical que determina o nível de atuação sobre bactérias, seja gram-negativa ou positiva (Yang et al., 2015).

\subsection{Extratos Vegetais}

Os extratos vegetais são quaisquer partes oriundas das plantas, que passam por processo de desidratação, moagem e trituração. Seu uso está normalmente voltado nas ações nutracêuticas presentes nos vegetais podendo trazer ao animal algum benefício através dos princípios ativos presentes nas frações da planta (Santos et al., 2013).

Tabela 1. Algumas das espécies vegetais utilizadas como aditivos fitogênicos.

\begin{tabular}{|c|c|c|c|c|}
\hline Espécie & Nome Científico & $\begin{array}{l}\text { Parte da } \\
\text { Planta }\end{array}$ & Princípio Ativo & Propriedades \\
\hline Alecrim & Rosmarinus officinalis & Folha & Cineol & Antioxidante \\
\hline Alho & Allium sativum L. & Bulbo & Alicina & Antimicrobiano \\
\hline Canela & Cinnamomum verum & Casca & Cinamaldeído & $\begin{array}{l}\text { Antisséptico, Estimulante } \\
\text { do TGI }\end{array}$ \\
\hline Cravo & Syzygium aromaticum & Semente & Eugenol & Antioxidante \\
\hline Gengibre & Zingiber officinale & Rizoma & Zingerol & Antibacteriano \\
\hline Hortelã & Mentha piperina & Folha & Mentol & Antioxidante \\
\hline Orégano & Origanum vulgare & Folha & Carvacrol & $\begin{array}{l}\text { Estimulante do TGI, } \\
\text { Antibacteriano }\end{array}$ \\
\hline $\begin{array}{l}\text { Pimenta } \\
\text { Vermelha }\end{array}$ & Capsicum annum & Fruto & Capsaicina & Estimulante TGI \\
\hline Tomilho & Thymus vulgaris $L$. & Planta & Timol & Antioxidante \\
\hline
\end{tabular}

Fonte: Adaptado de Menten (2002).

As vantagens da utilização dos extratos se devem ao fato de gerarem compostos novos nos animais que são incapazes de serem inativados por agentes patógenos, não oferecendo risco de toxicidade ao consumidor e detendo rápida degradação ambiental. As principais desvantagens estão em apresentar baixa estabilidade dos compostos orgânicos e não monitorar substancias tóxicas que possam aparecer nas soluções, além disso, possuem uma rápida degradação na presença de luz e calor, curta viabilidade, e dependência direta da metodologia de extração aplicada (Potenza, 2004).

\section{Substâncias Bioativas}

As substâncias bioativas (Figura 1) têm sido amplamente estudadas devido aos diversos efeitos extra nutricionais que promovem no organismo dos animais, entre eles o mais estudado é o potencial antioxidante promovido por estes alimentos que é dependente do tamanho, número e conformação de grupos hidroxila. 
Figura 1. Diagrama das substancias bioativas.

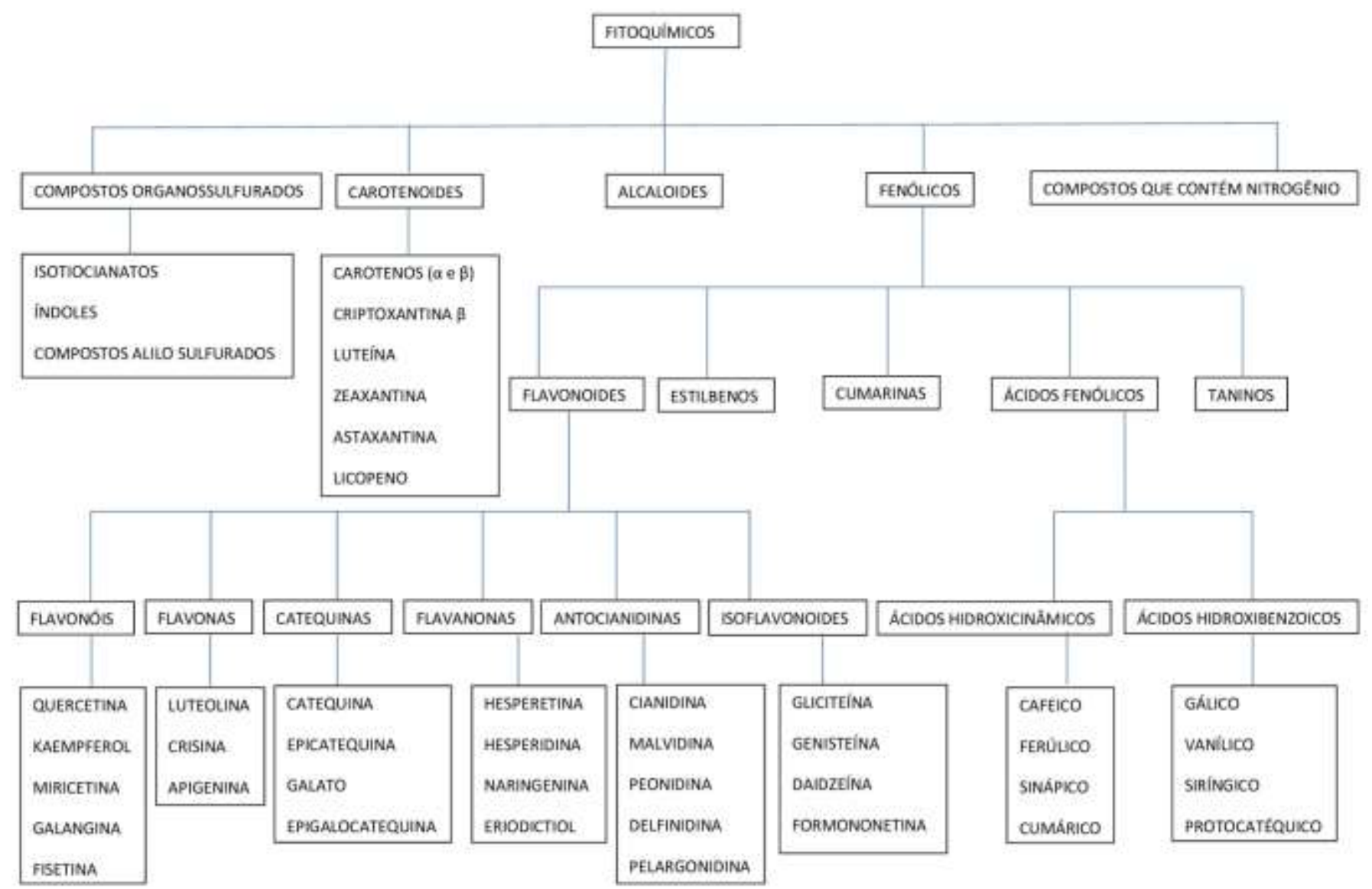

Fonte: Adaptado de Liu (2004).

Dentre estas substâncias estão os compostos fenólicos que representam a maior parte dos compostos bioativos. Eles são divididos de acordo com a cadeia carbônica em: flavonoides e não flavonoides. Os flavonoides são constituídos pelos flavanóis, isoflavonas, flavanonas, flavonóis (ou flavan-3-óis), flavanóis e antocianinas. Já os não flavonoides incluem fenóis simples, ácidos fenólicos, estilbenos entre outros (Pereira \& Cardoso, 2012).

Estes compostos doam elétrons reduzindo os efeitos dos radicais livres que podem acometer o organismo, tornando as células mais estáveis e menos propensas a oxidação (Shahidi \& Ambigaipalan, 2015). Atuam também sobre a resposta antiinflamatória ou modificando o DNA através do aumento da síntese de enzimas antioxidantes ou de forma terapêutica. Alguns exemplos desses compostos são as vitaminas (A, C, E), minerais (Zinco, Magnésio, Cobre, Selênio, Manganês), Fibras insolúveis e solúveis, polifenóis, carotenoides, fitoesteróis, compostos organossulfurados entre outros.

\subsection{Carotenoides}

Os carotenoides são os principais corantes naturais dentre os existentes na natureza (clorofilas, antocianinas e betaninas) e conferem as cores (Amarelo, Laranja e Vermelha) aos alimentos podendo exercer funções biológicas sobre a saúde (Martins et al., 2016; Rodriguez-Amaya, 2016). Na natureza são encontrados em seres vivos, entretanto apenas bactérias, fungos, algas e plantas superiores são capazes de produzir estes pigmentos.

Segundo Bogacz-Radomska (2018), esses pigmentos estão divididos entre Carotenos $\alpha$ e $\beta$, Luteína, Zeaxantina, Astaxantina, Licopeno e Criptoxantina $\beta$, e suas funções na alimentação se dão devido as suas características antioxidantes e precursores da vitamina A (Licopeno, Criptoxantina $\beta$, Carotenos $\alpha$ e $\beta$ ). 


\subsection{1 $\beta$-caroteno}

O beta caroteno é o mais comumente encontrado na natureza, a isso se dá devido a grande presença dele em frutas e verduras (Jeyakodi, Krishnanakumar \& Chellapan., 2018). Dentre os carotenóides precursores da vitamina A, o $\beta$-caroteno é que mais se destaca por gerar duas moléculas de retinol, enquanto que os demais geram apenas um (Bogacz-Radomska \& Harasym, 2018). Essa vitamina contribui para a manutenção do sistema imune, evita doenças cardiovasculares, protege contra os radicais livres com função antioxidante e atua no funcionamento ocular, mantendo a integridade da retina, que trabalha na fotorecepção e visão noturna (Jeyakodi et al., 2018). Além disso, ajuda na prevenção de uma doença chamada Protoporfiria Eritropoiética que é causada pela sensibilidade ao sol (Wahlin et al., 2011).

Apesar de possuir maior ação pró-vitamina A que os outros carotenoides, sua absorção e biodisponibilidade no organismo são reduzidos, graças às frações fibrosas da parede celular das plantas que retêm uma grande parcela, diminuindo assim a absorção, degradação e aproveitamento (Donhowe \& Kong, 2014).

\subsubsection{Licopeno}

O licopeno é um dos principais carotenoides encontrados na natureza e um dos mais abundantes no organismo dos animais, pois são oriundos dos alimentos que possuem tal pigmento em alta concentração. Entretanto, a sua biodisponibilidade é dependente da alimentação que acompanha este carotenoide, de forma que fatores como excesso e tipo de gordura, fibras, e processamento dos alimentos fontes, assim como outros carotenoides como a luteína e betacaroteno podem afetar o processo de absorção e de utilização através da competição na incorporação dos quilomicrons do intestino (Moritz \& Tramonte, 2006).

Dentre as funções do licopeno no organismo é possível mencionar que entre os carotenoides ele possui a maior capacidade antioxidante, mediante sequestro de oxigênio e protegendo moléculas de lipídio, VLDL, proteínas e DNA contra o processo oxidativo (Volp et al., 2011). Além disso, são relatados efeitos moduladores no metabolismo lipídico reduzindo a possibilidade de formação de placas de ateroma, pois apresenta característica redutora de colesterol através da diminuição de uma enzima produtora de colesterol de nome HMG-coa redutase (Palozza et al., 2010).

\subsection{Compostos Fenólicos}

Os compostos fenólicos originam-se do metabolismo das plantas tendo papel importante no crescimento, pigmentação, proteção contra patógenos e na reprodução, além de se formarem mediante a aparição de ferimentos na planta e também sob radiação solar. Já nos alimentos, conferem aroma, adstringência, cor e estabilidade oxidativa (Angelo \& Jorge, 2007). Dentre os fenóis estão os flavonoides, ácidos fenólicos, fenóis simples, cumarinas, taninos, ligninas, estilbenos entre outros (Angelo \& Jorge, 2007).

Dentre os compostos fenólicos estão aqueles que são conhecidos como fatores anti-nutricionais, que são produtos originados de vegetais e atuam como "células de defesa" dos vegetais, diminuindo sua digestibilidade e induzindo a um estado de recusa alimentar dos animais devido ao gosto ou até mesmo por ações danosas ao trato gastrointestinal (Souza et al., 2019). Apesar dos fatores anti-nutricionais terem uma má fama devido a todos os efeitos adversos que podem causar nos animais, nem tudo é ruim, pois dentre os mais conhecidos (Tanino, Ligninas e Gossipol) ainda são capazes de realizar possíveis efeitos benéficos aos animais (Souza et al., 2019).

Alguns pesquisadores têm recentemente utilizado alimentos com altas concentrações de taninos condensados para diferentes espécies, e relatado efeitos anti-helmínticos no controle de microrganismos (Arroyo-Lopez et al., 2014; Hoste et al., 2015). Para coelhos sabe-se que a Eimeria pode ser mortal, principalmente para láparos após os 20 dias (Pakandi, 2009), além de favorecer o aparecimento de outras doenças que podem acontecer simultaneamente (Duszynski \& Couch, 2013). 
Legendre et al. (2018) ao tentarem diminuir a concentração de microrganismos nas fezes dos animais, utilizaram dietas enriquecidas com taninos condensados a base de vagem de alfarroba e feno de sainfoin, 0,91 e 1,22\% respectivamente, para coelhos em fase de pré (0-34 dias) e pós desmama (35-101 dias) e observaram que houve uma redução de aproximadamente $60 \%$ das espécies de Eimeria com a utilização do feno durante todo o período pós desmama sem comprometer o desempenho dos animais.

Outros trabalhos que usaram taninos foram feitos e têm sido unanime entre eles que causa uma melhora sobre a digestibilidade da proteína microbiana (Min et al., 2005), além disso atuam como antioxidantes naturais (Zhang et al., 2010) e também como potentes inibidores de putrefação no processo de curtimento de couro (Naumann et al., 2017). Outro efeito foi relatado por Gai et al. (2009) que ocorreram reduções nos valores de TBARS com a adição de $0,5 \%$ na dieta.

A lignina tem se mostrado um fator limitante na utilização de forragens para animais, mas apesar disso Bezerra (2019) ao utilizar lignina purificada encontrou uma grande ação antioxidante da carne de ovinos em prateleira. Já o gossipol é um composto fenólico perigoso em sua forma livre, podendo causar sérios danos aos animais como edemas pulmonares, hipertrofia hepática, necrose muscular cardíaca, perda de apetite e problemas reprodutivos (Lima Júnior et al., 2010). Segundo Talipov et al. (2009), o gossipol apesar de ser altamente tóxico na forma livre possui importantes atividades antifúngicas, antivirais e anticancerígenas.

\subsection{Flavonoides}

Dentre os grupos fenólicos mais encontrados na natureza, os flavonoides são os mais diversificados, estando nas frutas, vegetais, cascas de arvores, sementes entre outros (Nijveldt et al., 2001). Os flavonoides são bastante conhecidos devido ao seu poder antioxidante (Fernandes et al., 2019), além disso foram mencionados efeitos anti-inflamatórios, antialérgicos, anticancerígeno entre diversos outros efeitos comprovados por pesquisas in vivo e in vitro. Esses compostos possuem diversas subclasses diferentes, entre elas estão: 3-flavanóis, flavanonas, flavonas, flavonóis, flavanas, isoflavonoides, chalconas, antocianidinas e isoflavonas.

Os flavonoides estão presentes em vários vegetais e frutas, entre eles podem ser citados: frutas vermelhas, frutos cítricos, legumes, verduras, chás, uvas, cereais e especiarias, dessa forma a obtenção de tais compostos são obtidos, exclusivamente, através da alimentação, pois os organismos animais não são capazes de sintetizar (Peterson et al., 1998).

\section{Fitogênicos na Reprodução}

A reprodução nos sistemas de produção dos animais possui fundamental importância, pois do ponto de vista econômico é de onde terá a produção de filhotes, que poderão repovoar o plantel ou serem comercializados vivos para Pets ou para produção de carne.

O estresse em coelhos é uma das principais causas de radicais livres no organismo, onde esses podem causar efeitos negativos sobre a capacidade reprodutiva dos coelhos. Dessa forma a utilização de alimentos que possam evitar o aparecimento dos radicais livres ou minimizar mostra-se fundamental, pois seus efeitos em machos podem acometer problemas na espermatogênese, acarretando em espermatozoides desformes ou mesmo diminuir a sua motilidade, acarretando a diminuição da chegada até o óvulo (El-Desoky et al., 2017). Já nas fêmeas observa-se que o estresse afeta diretamente a fertilidade, pois diminui os níveis de GnRH no organismo feminino privando os folículos do suporte de gonadotrofinas, impedindo assim que ocorra a ovulação.

Um dos compostos fitogênicos que vem sendo utilizada para coelhos é a moringa, por apresentar efeitos antioxidantes, que podem reduzir a produção de radicais livres e melhorar a fertilidade desses animais. Singh et al. (2009) 
relataram a presença de flavonóides como kaempferol, quercetina, vanilina e vários ácidos fenólicos, dentre eles os ácidos gálico, ferúlico, elágico e clorogenico.

Ewuola et al. (2015), utilizando coelhas da raça Nova Zelândia Branco e Chinchila com peso médio entre 2 e 2,5kg, avaliaram os efeitos da concentração crescente de extrato de folha de moringa crua $(0 ; 100 ; 200$ e $300 \mathrm{ml} / \mathrm{L})$ sobre os parâmetros sanguíneos e bioquímicos da gestação e lactação destes animais e observaram que qualquer um dos tratamentos utilizados aumentaram o volume sanguíneo, que está ligado a uma maior produção leiteira; reduziu linearmente a quantidade de urina e isso está correlacionado com uma maior limpeza dentro do ninho, visto que algumas coelhas urinam e defecam dentro do mesmo; aumentaram a quantidade de plaquetas circulantes no sangue, além de terem reduzido linearmente o teor de colesterol total dos animais.

El-Desoky et al. (2017) avaliarem diferentes concentrações de extrato aquoso de moringa (50;100;150 mg/kg PV), e constataram que mesmo em um período quente a utilização da moringa resultou em um crescimento linear para a concentração espermática, volume total do ejaculado, motilidade e funcionalidade total da fração do sêmen, sobrevivência, menor quantidade de espermatozoides anormais (incluindo cabeça e cauda), além de ter garantido a integridade do acrossoma e da membrana celular do espermatozoide.

Os autores justificam nos resultados obtidos que apesar do estresse por calor destruir as células de Leydig, diminuir a quantidade de testosterona e atrasar a espermatogênese, não foi observado tal efeito, de forma que houve uma melhoria na síntese da testosterona, além da própria atividade antioxidante oriunda da moringa que atuou protegendo os espermatozoides contra a oxidação. Os mesmos atribuem os resultados encontrados também à presença de ácidos graxos presentes na composição da moringa, especialmente os ácido $\alpha$-linolênico (C18:3n-3) e ao ácido dihomo $\gamma$-linoleico (DHLA; C20:3n-6), pois atuam provendo fluidez a membrana do espermatozoide, troca de íons e motilidade.

Outro fitogênico utilizado na alimentação de coelhos visando efeitos positivos sobre a reprodução são as folhas de tomilho, onde Ahmed et al. (2020) ao utilizarem diferentes níveis de inclusão destas folhas $(0 ; 4 ; 8 ; 12$ e 16g/kg) para coelhos em crescimento sob climas quentes, observaram que houve um resultado quadrático crescente a medida que houve aumento da concentração de folhas na alimentação no volume do sêmen, na taxa de sobrevivência dos espermatozoides, na motilidade, enquanto que houve também uma diminuição linear das anormalidades nos espermatozoides.

\section{Substâncias Bioativas na Reprodução}

A infertilidade é algo comum que pode acontecer com os animais de produção, gerando prejuízos aos produtores. Na busca de melhorar a fertilidade dos animais vem sendo utilizados substâncias bioativas que tem função de estimular a viabilidade, motilidade e sobrevivência dos espermatozoides (Abdelnour et al., 2020a).

Uma dessas substâncias bioativas utilizados na alimentação animal visando melhorar os parâmetros reprodutivos é a geleia real (Alagawany et al., 2019). Esse alimento é extremamente benéfico por apresentar em sua composição às vitaminas do complexo B, proteínas e minerais e diversos compostos biativos acetilcolina, prolactina, adenosina, polifenóis e flavonoides (Maghsoudlou et al., 2019).

Dentre os trabalhos na literatura que têm sido relatados com a inclusão da geleia real na alimentação de coelhos, verificou-se que quantidades variando de 50 a $150 \mathrm{mg}$, fornecidas $2 \mathrm{x}$ ao dia, garantiu aumento nos resultados de 10 a $26 \%$ de motilidade, de 10 a $20 \%$ de concentração espermática e de 5 a $11 \%$ de fertilidade, além de ter reduzido entre 14 e $30 \%$ a mortalidade dos espermatozoides (El-Hanoun et al., 2014).

Outro fator importante na reprodução é a qualidade dos espermatozoides, devido a composição ser formada por uma camada lipídica que age como um componente funcional e estrutural da célula espermática, protegendo e nutrindo a célula durante o trajeto até o óvulo. 
A camada lipídica é formada por uma grande quantidade de ácidos graxos PUFA da série n-6, e um baixo teor n-3, nos coelhos, que diferem assim da maioria dos mamíferos, que essa quantidade é exatamente ao contrário (Gliozzi et al., 2009). Isso mostra que o sémen dos coelhos por natureza é mais facilmente protegido contra oxidações devido à insaturações das cadeias de ácidos graxos. O número de insaturações na cadeia aumenta a peroxidação dos espermatozoides, conferindo uma característica negativa ao liquido seminal, tendo assim espermatozoides de baixa qualidade e uma maior mortalidade (Bansal \& Bilaspuri, 2011).

Esses efeitos são combatidos com antioxidantes que agem reduzindo o estresse oxidativo e melhorando a motilidade e sobrevivência. Dentre os antioxidantes os mais conhecidos são os carotenoides, vitaminas (E e C), selênio entre outros (Agarwal et al., 2004).

Outro pigmento que tem sido utilizado na reprodução de coelhos é o licopeno. Presente em um número limitado de frutos laranja-avermelhados (melancia, tomate, goiaba, mamão e pitanga) é classificado como carotenoide, e como tal apresenta um grande potencial como antioxidante.

Dessa forma Mangiagalli et al. (2012) utilizaram o licopeno em dois diferentes níveis de inclusão $(0,1$ e $0,5 \mathrm{mg} / \mathrm{L})$ na água para coelhos em crescimento e observaram que houve aumento no volume de sêmen ejaculado e no número de espermatozoides total por $\mathrm{ml}$ no espermatozoide fresco e após 24 horas de resfriamento a $4^{\circ} \mathrm{C}$, houve um atraso na oxidação dos espermatozoides resultando em melhora da motilidade e a viabilidade no nível de 0,5mg/L.

\section{Fitogênicos no Bem Estar}

O bem estar dos animais tem sido uma forte preocupação por parte dos produtores, que buscam cada vez mais oferecer aos seus animais melhores instalações e manejos. Uma das maiores preocupações na cunicultura é a saúde intestinal dos animais, pois a presença da microbiota cecal é extremamente importante para os coelhos de forma que estes microrganismos produzam energia, proteínas e vitaminas. A saúde do trato gastrointestinal desses animais garante o controle sobre a população microbiana existente, e essa relação com o hospedeiro é um importante componente do sistema imune dos animais. Segundo Kirjavainen e Gibson (1999), algumas dessas são bactérias benéficas, apatogênicas e protetoras da mucosa intestinal, quando em desbalanço, podem acarretar disbioses severas ou até mesmo o óbito dos animais.

Um dos produtos recentemente pesquisados para coelhos com potencial anti-inflamatório e benéfico para a microbiota cecal é a planta Boswellia serrata (BS). Ismail et al. (2019), ao utilizarem diferentes níveis da folha da BS ( $0 ; 0,25$; 0,$5 ; 0,75 ; 1 \mathrm{~g} / \mathrm{kg}$ dieta) para coelhos em crescimento, observaram que houve uma redução significativa do total de bactérias patogênicas e Salmonela para todos os tratamentos testes utilizados, enquanto que para $E$. coli apenas o tratamento de 0,75 não diferiu do grupo controle, mas numericamente houve diminuição dos valores.

Outro vegetal estudado para coelhos é a Moringa oleífera, que além de exercer funções no sistema cardíaco, ainda atua como anti-inflamatório, bactericida e antioxidante, de forma que qualquer parte da planta (folhas, raízes, sementes, fruto, flores) é capaz de exercer algumas destas funções. Em um desses trabalhos, El-Badawi et al. (2017), utilizando níveis crescentes do pó da folha da moringa $(0 ; 0,5 ; 1 \%)$ para coelhos em crescimento, observaram que houve expressiva redução da quantidade de bactérias patogênicas de $33 \times 10^{4} \mathrm{cfu} / \mathrm{ml}$ do grupo controle para 2,37 e 5,27x $10^{4} \mathrm{cfu} / \mathrm{ml}$, para os tratamentos que continham 0,5 e $1 \%$ do pó de moringa, respectivamente. Além desse resultado, os autores relataram a diminuição da quantidade de Staphylococcus aureus de $89 \times 10^{2} \mathrm{cfu} / \mathrm{ml}$ para 0,77 e $0,50 \times 10^{2} \mathrm{cfu} / \mathrm{ml}$ para os tratamentos que continham 0,5 e $1 \%$ do pó de moringa, respectivamente. Os autores justificam que a moringa possui diversos compostos, dentre eles estão os ácidos graxos de cadeia curta que atuam combatendo as bactérias patogênicas enquanto promovem a proliferação das bactérias boas que atuam no sistema de defesa do organismo. Na parte histológica foi observado que a adição dos tratamentos com 
moringa melhorou significativamente o comprimento do vilo e a profundidade da cripta do jejuno dos coelhos, esses resultados refletem em uma melhor absorção do nutriente e da digestibilidade do alimento.

Hashem et al. (2019) utilizaram extratos etanólicos da folha e da raiz para coelhos em crescimento e observaram que para as variáveis sanguíneas apenas o extrato da raiz foi capaz de melhorar o teor de plaquetas e hemoglobinas, já para os metabólitos plasmáticos houve aumento em ambos para a albumina, proteína essa de extrema importância no processo de absorção de ácidos graxos de cadeia curta e média. Já no que se refere aos indicadores antioxidantes, constatou-se que ambos os extratos aumentaram significativamente a capacidade antioxidante total, diminuindo assim as reações de peroxidação lipídica no organismo, enquanto reduziram os teores de malondialdeido nos animais, garantindo assim uma menor quantidade de radicais livres na carcaça dos animais.

\section{Substâncias Bioativas no Bem Estar}

A utilização de alimentos com funções nutracêuticas na alimentação animal já é bastante comum em outras culturas animais, entretanto este tema para coelhos tem tido uma maior atenção nos últimos anos, devido às substâncias que se fazem presentes na composição dos ingredientes, que causam efeitos positivos no bem-estar dos animais, nos parâmetros sanguíneos, assim como na microbiota intestinal.

Segundo Bazzano, Serdula e Liu. (2003), a alimentação pode influenciar diretamente a saúde de quem está ingerindo o alimento, de forma que a ingestão de frutas legumes, grãos, óleos e especiarias devem ser consumidos adequadamente e com frequência. Pois um dos principais locais de atuação é no sistema cardiovascular ajudando na prevenção de doenças cardíacas, assim como na prevenção de aterosclerose, que forma placas de ateroma bloqueando a passagem por veias e artérias com núcleos necróticos, regiões calcificadas, lipídios modificados acumulados, células musculares lisas inflamadas, células endoteliais, leucócitos, e células espumosas.

Visando diminuir tais efeitos sobre o sistema cardíaco de coelhos, Bhaskar et al. (2013) inseriu na alimentação a Quercetina por 90 dias e comparou com dietas hipercolesterolêmicas e observaram que este inibiu a atuação de enzimas inflamatórias no organismo (5-LPX, 12-LPX, COX, SON, MPO), assim como reduziu significativamente um biomarcador específico que avalia doenças cardíacas de nome CRP. Quanto à aterosclerose, foi observada uma significativa redução com rações hipercolesterolêmicas, e foi justificado pelos autores que a Quercetina possui um potencial antioxidante e antinflamatório.

Buscando encontrar formas alternativas para melhorar o bem-estar dos animais, Abdelnour et al. (2020b), utilizaram um complexo proteína-pigmento, de nome ficocianina, para coelhos em crescimento e avaliando diferentes níveis de inclusão $(0 ; 50 ; 100$ e $150 \mathrm{mg} / \mathrm{kg}$ dieta) sobre as variáveis sanguíneas e a microbiota cecal observaram que para os parâmetros do sangue houve em aumento da quantidade de hemácias nos tratamentos de 50 e $100 \mathrm{mg}$, no número de plaquetas no tratamento de $50 \mathrm{mg}$ e aumento na hemoglobina para os três tratamentos. Já no que se refere a microbiota cecal avaliado pelos autores, foi observado que houve diminuição das bactérias: E. coli, Salmonella e Enterococci para todos os tratamentos testes utilizados. Esses resultados podem ter ocorrido devido à ficocianina possui em sua composição propriedades capazes de atuar de forma antioxidante, anti-inflamatória e modulatória do sistema imune (Farag et al., 2016; Osman et al., 2019; Xie et al., 2019).

\section{Fitogênicos na Qualidade da Carne}

As ervas e especiarias utilizadas na cunicultura têm sido componentes importantes no processo de defesa antioxidante da carne, retardando efeitos negativos sobre a deterioração de gordura e proteína, e mantendo o sabor, cor, valor nutritivo e tempo de prateleira, através da presença de compostos como os flavonoides, taninos, ácidos fenólicos, vitaminas A, C e E. 
Além do potencial antioxidante, também são utilizados para prolongar o tempo de prateleira das carnes e a integridade do tecido dos animais. Alguns desses alimentos são inseridos de formas diferentes, as ervas e especiarias (Orégano, Rosemary, Própolis, Erva-doce, Rooibos, Tomilho, Sumagre entre outros) e os extratos também podem ser adicionados aos produtos cárneos durante e após os processamentos com o intuito de garantir uma maior durabilidade dos nutrientes e do alimento (Dalle Zotte, Celia \& Szendro, 2016).

O orégano pode ser suplementado na forma de óleo ou de extrato (Cardinali et al., 2015), em ambas por conter propriedades antimicrobianas e antioxidantes (monoterpenos, carvacrol e timol) foi observada uma significativa melhora sobre a estabilidade oxidativa da carne, além de aumentar o tempo de prateleira de carnes de coelhos refrigeradas por até 12 dias.

Dalle Zotte et al. (2014) utilizando níveis de extrato de chá de Rooibos fermentado (0,5; 1 e 2\%) em hambúrgueres de carne de coelho por 6 dias observaram que todos os níveis foram capazes de diminuir a peroxidação quando comparados com os tratamentos que não receberam o produto, além disso constatou-se também que o nível de 0,5\% foi bem aceito sensorialmente pelos consumidores.

Ali et al. (2015), visando estender o tempo de prateleira da carne de coelho sob refrigeração e estocagem por 15 dias, avaliaram os efeitos de $0,5 \%$ de ácido lático, $0,5 \%$ de óleo de tomilho e $8 \%$ de extrato aquoso de sumagre sob mergulho por 1 , 1 e 10 minutos, respectivamente. Os autores observaram que a utilização do sumagre e do ácido lático conferiram ao hambúrguer um maior tempo de prateleira por 3 e 6 dias sob refrigeração de $2^{\circ} \mathrm{C}$, respectivamente. Ainda justificaram que o ácido lático e o sumagre evitaram a proliferação de bactérias na carne por mais tempo que o óleo de tomilho, esse resultado foi capaz de influenciar na análise sensorial visto que cor e odor já não estavam agradando aos consumidores nos últimos dias.

\section{Substâncias Bioativas na Qualidade da Carne}

As substâncias bioativas têm sido bastante utilizadas na alimentação animal com o intuito de melhorar a qualidade da carne. Dentre estes compostos, os mais utilizados são os antioxidantes, visto que a carne de coelhos possui uma grande quantidade de ácidos graxos polinsaturados (PUFAS) que são mais suscetíveis à oxidação lipídica, diminuindo o tempo de prateleira através da rancidez e da deterioração da cor (Dalle Zotte \& Szendro, 2011).

Corino et al. (2007) avaliando a associação do ácido linoleico conjugado (CLA) nos níveis de 0 e 0,5\% e da vitamina E nas concentrações de 60 e 240mg/kg de dieta para coelhos em crescimento, observaram que não houve efeito da inclusão nem da interação para desempenho, entretanto o nível de $240 \mathrm{mg}$ de vitamina E resultou em maiores peso ao abate e de carcaça, aumentou os teores de ácidos graxos monoinsaturados (MUFAS) e reduziu as PUFAS, que segundo os autores são resultados positivos, pois sua redução diminui as chances da carne sofrer oxidação, visto que as cadeias PUFAS estão mais propensas a sofrer quebras do que as MUFAS gerando maiores radicais livres. Já no que se refere à estabilidade oxidativa, foi possível observar que o nível de $60 \mathrm{mg}$ de vitamina $\mathrm{E}$ resultou em um alto valor de substancias reativas ao ácido tiobarbitúrico (TBARS) de $(1300 \mathrm{mg} / \mathrm{kg}$ ) resultado bem superior ao de $240 \mathrm{mg} / \mathrm{kg}$ que apresentou um valor de 650 no TBARS.

O nível de oxidação e produção de radicais livres é maior com o aumento das insaturações dentro da cadeia, produzindo até 25 vezes mais peróxidos e radicais livres com PUFAS (ácido linolênico, 18:3) quando comparadas com MUFAS (ácido oleico, 18:1) e de até 100 vezes o consumo de oxigênio (TOZER, 2001).

Ebeid et al. (2013) utilizando vitamina E, selênio orgânico e a interação entre os dois produtos para coelhos em crescimento relataram que todos os tratamentos testes melhoraram o peso final, ganho de peso diário, conversão alimentar e o peso de carcaça quente. Quanto a qualidade da carne os tratamentos testes foram superiores ao grupo controle, mas não diferiram entre si apresentando 40 e 74\%, menos reações com o TBARS do que o grupo controle para 3 e 6 dias, respectivamente. 


\section{Considerações Finais}

A utilização de aditivos fitogênicos na produção de coelhos representa uma ferramenta importante para a substituição dos antimicrobianos melhoradores de desempenho por fontes naturais e que traz benefícios para o animal e o consumidor. Muitas pesquisas já demostram que esses produtos alternativos são a alternativa mais viável para a produção. Diversas plantas possuem substâncias bioativas que podem ser utilizados como suplementos alimentares multifuncionais para os coelhos. Isso pode ser observado com a inclusão de ervas e óleos essenciais que possuem diversas funções sobre o desempenho, a atividade antimicrobiana, benefícios na saúde intestinal, prevenção da oxidação lipídica e melhoras na reprodução.

\section{Referências}

Abdelnour, S. A., Abd El-Hack, M. E., Alagawany, M., Taha, A. E., Elnesr, S. S., Abd Elmonem, O. M. \& Swelum, A. A. (2020a). Useful impacts of royal jelly on reproductive sides, fertility rate and sperm traits of animals. Journal of Animal Physiology and Animal Nutrition, 104 (6), $1798-1808$.

Abdelnour, S. A., Swelum, A. A., Salama, A., Al-Ghadi, M. Q., Qattan, S. Y. A., Abd El-Hack, M. E., Asmaa, F., Khafaga, A. R., Alhimaidi, B. O., Ammari, A. A. \& El-Saadony, M. T. (2020b). The beneficial impacts of dietary phycocyanin supplementation on growing rabbits under high ambient temperature. Italian Journal of Animal Science, 19(1), 1046-1056.

Agarwal, A., Nallella, K. P., Allamaneni, S. R. \& Said, T. M. (2004). Role of antioxidants in treatment of male infertility: an overview of the literature. Reproductive BioMedicine, 8, 616-627.

Ahmed, A. E., Alkahtani, M. A., Aboubaker, A. \& Abdel-Wareth, A-M. (2020). Thyme leaves as an eco-friendly feed additive improves both the productive and reproductive performance of rabbits under hot climatic conditions. Veterinární Medícina, 65(12), 553-563.

Alagawany, M., Elnesr, S. S., Farag, M. R., Abd El-Hack, M. E., Khafaga, A. F., Taha, A. E., Tiwari, R., Yatoo, M. I., Bhatt, P., Gopi, M. \& Kuldeep, D. (2019). Use of Licorice (Glycyrrhiza glabra) Herb as a Feed Additive in Poultry: Current Knowledge and Prospects. Animals, 9(8), 536-546.

Ali, F. H. M., Hassan, A. R. H. A. \& Bahgat, A. E. M. (2015). Study of sumac extract (Rhus coriaria L.), lactic acid and thyme oil as decontaminants for shelf life extension of refrigerated rabbit meat. Food Science and Quality Management, 38, 71-78.

Angelo, P. M. \& Jorge, N. (2007). Compostos fenólicos em alimentos - uma revisão. Revista do Instituto Adolfo Lutz, 66(1), 1-9.

Arroyo-Lopez, C., Manolaraki, F., Saratsis, A., Saratsi, K., Stefanakis, A., Skampardonis, V., Voutzourakis, N., Hoste, H. \& Sotiraki, S. (2014). Anthelmintic effect of carob pods and sainfoin hay when fed to lambs after experimental trickle infections with Haemonchus contortus and Trichostrongylus colubriformis. PUBMED, 21, 1-9.

Bansal, A. K. \& Bilaspuri, G. S. (2011). Impacts of oxidative stress and antioxidants on semen functions. Veterinary Medicine International, $1-7$.

Bazzano, L. A., Serdula, M. K. \& Liu, S. (2003). Dietary intake of fruits and vegetables and risk of cardiovascular disease. Current Atherosclerosis Reports, 5 , 492-499.

Bezerra, H. V. A. (2019). Lignina purificada na dieta de ruminantes: impacto no desempenho e saúde de ovinos. 75 f. Dissertação (Mestrado) - Curso de Zootecnia, Universidade de São Paulo, Pirassununga.

Bhaskar, M. P. S., Kumar, K. S., Krishnan, M. P. K. \& Antony, H. (2013). Quercetin alleviates hypercholesterolemic diet induced inflammation during progression and regression of atherosclerosis in rabbits. Nutrition, 29, 219-229

Bogacz-Radomska, L. \& Harasym, J. (2018). $\beta$-Carotene-properties and production methods. Food Quality and Safety, 2(2), 1-6.

Brasil. Ministério da Agricultura, Pecuária e Abastecimento. Mapa. Instrução Normativa Mapa no 13, de 01 de dezembro de 2004 , sobre a regulamentação técnica dos aditivos para produtos destinados a alimentação animal.

Cardinali, R., Cullere, M., Dal Bosco, A., Mugnai, C., Ruggeri, S., Mattioli, S., Castellini, C., Marinucci, M.T \& Dalle Zotte, A. (2015). Oregano, Rosemary and Vitamin E dietary supplementation in growing rabbits: effect on growth performance, carcass traits, bone development and meat chemical composition. Livestock Science, 175, 83-89.

Cardoso, V. S., Lima, C. A. R., Lima, M. E. F., Dorneles, L. E. G. \& Danelli, M. G. M. (2012). Piperine as a phytogenic additive in broiler diets. Pesquisa Agropecuária Brasileira, 47, 489-496.

Cho, J. H., Kim, H. J. \& Kim, I. H. (2014). Effects of phytogenic feed additive on growth performance, digestibility, blood metabolites, intestinal microbiota, meat color and relative organ weight after oral challenge with clostridium perfringens in broilers. Livestock Science, $160,82-88$.

Corino, C., Lo Fiego, D.P., Macchioni, P., Pastorelli, G., Di Giancamillo, A., Domeneghini, C. \& Rossi, R. (2007). Influence of dietary conjugated linoleic acids and vitamin E on meat quality, and adipose tissue in rabbits. Meat Science, 76(1), 19-28. 
Cruz, G. F. L., Xavier Neta, G. C.., Duarte, I. N. H.., Barbosa, R. V., Sousa, E. C. L., Azevedo, P. S., Costa, M. L. L., Panosso, N. M., Bessa, A. F. O., Fernandes, L. S., Borges, P. \& Araújo, L.S. (2020). Carcass traits and meat quality of Lionhead rabbits. Research, Society and Development, 9(9), p. e736997887.

Dalle Zotte, A. \& Szendro, Z. (2011). The role of rabbit meat as functional food. Meat Science, 88(3), 319-331.

Dalle Zotte, A., Mousavikhorshidi, H., Pizzocchero, V. \& Cullere, M. (2014). Rooibos (Aspalathus linearis) is an effective functional ingredient in retarding lipid oxidation of cooked rabbit meat patties. In: Proceedings of the 60th International Congress of Meat Science and Technology. Punta del Este, Uruguay, poster 204.

Dalle Zotte, A., Celia, C. \& Szendro, Z. (2016). Herbs and spices inclusion as feedstuff or additive in growing rabbit diets and as additive in rabbit meat: A review. Livestock Science, 189, 82-90.

Donhowe, E.G. \& Kong, F. (2014). Beta-carotene: Digestion, Microencapsulation, and In Vitro Bioavailability. Food and Bioprocess Technology, 7(2), 338354.

Duszynski, D.W. \& Couch, L. (2013). The biology and identification of the Coccidia (apicomplexa) of rabbits of the world. 1st edition. Elsevier, Amsterdam. 352p.

Ebeid, T. A., Zeweil, H. S., Basyony, M. M., Dosoky, W. M. \& Badry, H. (2013). Fortification of rabbit diets with vitamin E or selenium affects growth performance, lipid peroxidation, oxidative status and immune response in growing rabbits. Livestock Science, 155(2), 323-331.

El-Badawi, A. Y., El-Wardany, I., El-Moez, S. I. A., Helal, F. I. S., Ali, N. G. M., Shourrap, M. I. \& Aboelazab, O. M. (2018). Impact of dietary Moringa oleifera leaves on intestinal pathogenic load and histological structure of growing rabbits raised under heat-stress conditions. Animal Production Science, 58(10), A-G.

El-Desoky, N. I., Hashem, N. M., Elkomy, A. \& Abo-elezz, Z. R. (2017). Physiological response and semen quality of rabbit bucks supplemented with Moringa leaves ethanolic extract during summer season. Animal, 11(9), 1549-1557.

El-Hanoun, A. M., Elkomy, A. E., Fares, W. A. \& Shahienl, E. H. (2014). Impact of royal jelly to improve reproductive performance of male rabbits under hot summer conditions. World Rabbit Science, 22(3), 241-248.

Ewuola, E. O., Sokunbi, O. A., Sanni, K. M., Oyedemi, O. M. \& Lawal, T. T. (2015). Haematological and serum biochemical responses of rabbit does to crude Moringa oleifera leaf extract at gestation and lactation. Tropical Animal Health and Production, 47(4), 637-642.

Farag, M. R., Alagawany, M., Abd El-Hac, M. E. \& Dhama, K. (2016). Nutritional and healthical aspects of Spirulina (Arthrospira) for poultry, animals and human. International Journal of Pharmacology, 12(1), 36-51.

Fernandes, R. C., Anami, J. M. \& Steffens, C. A. (2019). Maçã: compostos fenólicos e saúde. Revista interdisciplinar de estudos em saúde da UNIARP, 9(2), 29-33.

Gadde, U., Kim, W. H., Oh, S. T. \& Lillehoj, H. S. (2017). Alternatives to antibiotics for maximizing growth performance and feed efficiency in poultry: a review. Animal Health Research Reviews, 18, 26-45.

Gai, F., Gasco, L., Liu, H.W., Lussiana, C., Brugiapaglia, A. \& Masoero, G. (2009). Effect of diet Chestnut tannin supplementation on meat quality, fatty acid profile and lipid stability in broiler rabbits. Italian Journal of Animal Science, 8, 787-789.

Gliozzi, T. M., Zaniboni, L., Maldjian, A., Luzi, F., Maertens, L. \& Cerolini, S. (2009). Quality and lipid composition of spermatozoa in rabbits fed DHA and vitamin E rich diets. Theriogenology, 71, 910-919.

Gopi, M., Karthik, K., Manjunathachar, H. V., Tamilmahan, P., Kesavan, M., Dashprakash, M., Balaraju, B. L. \& Purushothaman, M. R. (2014). Essential oils as a feed additive in poultry nutrition. Advance in Animal and Veterinary Sciences, 2(1), 1-7.

Hashem, N. M., Soltan, Y. A., El-Desoky, N. I., Morsy, A. S. \& Sallam, S. M. A. (2019). Effects of Moringa oleifera extracts and monensin on performance of growing rabbits. Livestock Science, 228, 136-145.

Hoste, H., Torres-Acosta, J. F. J., Sandoval-Castro, C. A., Mueller-Harvey, I., Sotiraki, S., Louvandini, H., Thamsborg, S. M. \& Terrill, T. H. (2015). Tannin containing legumes as a model for nutraceuticals against digestive parasites in livestock. Veterinarian Parasitology, $212(2), 5-17$.

Ismail, I. E., Abdelnour, S. A., Shehata, S. A., Abd El-Hack, M. E., El-Edel, M. A., Taha, A. E., Schiavitto, M. \& Tufarelli, V. (2019). Effect of Dietary Boswellia serrata Resin on Growth Performance, Blood Biochemistry, and Cecal Microbiota of Growing Rabbits. Frontiers in Veterinary Science, 6, 1-8.

Jayasena, D. D. \& Jo, C. (2013). Essential oils as potential antimicrobial agents in meat and meat products: A review. Trends in Food Science \& Technology, 34(2), 96-108.

Jeyakodi, S., Krishnanakumar, A. \& Chellapan, D. K. (2018). Beta carotene therapeutic potential and strategies to enhance its bioavaliability. Nutrition \& Food Science, 7(4), 1-6.

Karásková, K., Suchý, P. \& Straková, E. (2015). Current use of phytogenic feed additives in animal nutrition: a review. Czech Journal of Animal Science, 60(12), 521-530.

Khattak, F., Ronchi, A., Castelli, P. \& Sparks, N. (2014). Effects of natural blend of essential oil on growth performance, blood biochemistry, cecal morphology, and carcass quality of broiler chickens. Poultry Science, 93, 132-137. 
Kirjavainen, P. V. \& Gibson G. R. (1999). Healthy gut microflora and allergy: factors influencing development of the microbiota. Annals of Medicine, 31, 288-292.

Koiyama, N. T. G. (2012). Aditivos fitogênicos na produção de frangos de corte. Animal Science. Universidade Federal de Santa Catarina, Florianópolis, SC.

Kutlu, T. \& Erdoðan, Z. (2010). In Poultry Feeding Phytobiotic Feed Additives. Coop Animals Congress, 07-09 October 2010, Kayseri.

Legendre, H., Saratsi, K., Voutzourakis, N., Saratsis, A., Stefanakis, A., Gombault, P., Hoste, H., Gidenne, T. \& Sotiraki, S. (2018). Coccidiostatic effects of tannin-rich diets in rabbit production. PUBMED, 117(12), 3705-3713.

Lima Júnior, D. M., Monteiro, P. D. B. S., Rangel, A. H. N., Maciel, M. V., Oliveira, S. E. O. \& Freire, D. A. (2010). Fatores anti-nutricionais para ruminantes. Acta Veterinaria Brasilica, 4(3), 132-143.

Liu, R.H. (2004). Potential synergy of phytochemicals in cancer prevention: mechanism of action. Journal of Nutrition, 134(12), 3479-3485.

Madhupriya, V., Shamsudeen, P., Manohar, G.R., Senthilkumar, S., Soundarapandiyan, V. \& Moorthy, M. (2018). Phyto feed additives in poultry nutrition-a review. International Journal of Science, Environment and Technology, 7(3), 815-822.

Maenner, K., Vahjen, W. \& Simon, O. (2011). Studies on the effects of essential-oil based feed additives on performance, ileal nutrient digestibility, and selected bacterial groups in the gastrointestinal tract of piglets. Journal of Animal Science, 89, 2106-2112.

Maghsoudlou, A., Mahoonak, A.S., Mohebodini, H. \& Toldra, F. (2019). Royal jelly: Chemistry, storage and bioactivities. Journal of Apicultural Science, $63(1), 17-40$.

Mangiagalli M. G., Cesari, V., Cerolini, S., Luzi, F. \& Toschi, I. (2012). Effect of lycopene supplementation on semen quality and reproductive performance in rabbit. World Rabbit Science, 20, 141-148.

Martins, N., Roriz, C. L., Morales, P., Barros, L. \& Ferreira, I. C. F. R. (2016). Food colorants: Challenges, opportunities and current desires of agro-industries to ensure consumer expectations and regulatory practices. Trends in Food Science \& Technology, 52, 1-15.

Menten, J. F. M. (2002). Probióticos, Prebióticos e Aditivos Fitogênicos na nutrição de aves. In: II Simpósio Sobre Nutrientes Na Alimentação Animal. Anais... Uberlândia, p. 251-276.

Min, B. R., Attwood, G. T., McNabb, W. C., Molan, A. L. \& Barry, T. N. (2005). The effect of condensed tannins from Lotus corniculatus on the proteolytic activities and growth of rumen bacteria. Animal Feed Science and Technology, 121(1), 45-58.

Moritz, B. \& Tramonte, V. L. C. (2006). Biodisponibilidade do licopeno. Revista de Nutrição, 19(2), 265-273.

Mueller, K., Blum, N.M., Kluge, H. \& Mueller, A.S. (2012). Influence of broccoli extract and various essential oils on performance and expression of xenobiotic-and antioxidant enzymes in broiler chickens. British Journal of Nutrition, 108(4), 588-602.

Naumann, H. D., Tedeschi, L. O., Zeller, W. E. \& Huntley, N. F. (2017). The role of condensed tannins in ruminant animal production: advances, limitations and future directions. Revista Brasileira de Zootecnia, 46(12), 929-949.

Nijveldt, R. J., Van Nood, E., Van Hoorn, D. E. C., Boelens, P. G., Van Norren, K. \& Van Leeuwen, P. A. M. (2001). Flavonoids: a review of probable mechanisms of action and potential applications. The American journal of clinical nutrition, 74, 418-425.

Osman, A., Abd-Elaziz, S., Salama, A., Eita, A. A. \& Sitohy, M. (2019). Health protective actions of phycocyanin obtained from an Egyptian isolate of Spirulina platensis on albino rats. EurAsia Journal of BioScience, 13, 105-112.

Pakandl, M. (2009). Coccidia of rabbit: a review. Parasitology, 56(3), 153-166.

Palozza, P., Parrone, N., Simone, R.E. \& Catalano, A. (2010). Lycopene in atherosclerosis prevention: an integrated scheme of the potential mechanisms of action from cell culture studies. PUBMED, 504(1), 26-33.

Pereira, R. J. \& Cardoso, M. D. G. (2012). Vegetable secondary metabolites and antioxidants benefits. Journal of Biotechnology and Biodiversity, 3, 146-152.

Peterson, J. D. (1998). Flavonoids: Dietary occurrence and biochemical activity. Nutrition Research, 18, 1995-2018.

Potenza, M. R. (2004). Produtos naturais para o controle de pragas. In: X Reunião Itinerante de Fitossanidade do Instituto Biológico: Café, 5., 2004, Mooca. Anais... São Paulo,SP, 2004. p.89-100.

Rodriguez-Amaya, D. B. (2016). Natural food pigments and colorants. Current Opinion in Food Science, 7, 20-26.

Santos, P. L., Prando, M. B., Morando, R., Pereira, G. V. N. \& Kronka, A. Z. (2013). Utilização de extratos vegetais em proteção de plantas. Centro Científico Conhecer - Goiânia, 9(17), 2562-2576.

Shahidi, F. \& Ambigaipalan, P. (2015). Phenolics and polyphenolics in foods, beverages and spices: Antioxidant activity and health effects - A review. Journal of Functional Foods, 18, 820-897.

Singh, B. N., Singh, B. R., Singh, R. L., Prakash, D., Dhakarey, R., Upadhyay, G. \& Singh,H. B. (2009). Oxidative DNA damage protective activity, antioxidant and anti-quorum sensing potentials of Moringa oleifera. Food and Chemical Toxicology, 47(6), 1109-1116. 
Research, Society and Development, v. 10, n. 12, e306101220103, 2021

(CC BY 4.0) | ISSN 2525-3409 | DOI: http://dx.doi.org/10.33448/rsd-v10i12.20103

Souza, C. G., Moura, A. K. B., Silva, J. N. P., Soares, K. O., Silva, J. V. C. \& Vasconcelos, P. C. (2019). Fatores anti-nutricionais de importância na nutrição animal: Composição e função dos compostos secundários. PUBVET, 13(5), 1-19.

Talipov, S. A., Mamadrakhimov, A. A., Tiljakov, Z. G., Dowd, M. K., Ibragimov, B. T. \& Xonkeldieva, M. T. (2009). The Crystal and Molecular Structure of Dianhydrogossypol. Journal of the American Oil Chemistry Society, 86(3), 207-213.

Tozer, K. N. (2001). Qualin improvement and shelf-life extension of fish filets from three aquaculture species. Dissertação (Mestrado) - University of Guelph. Ontário, Canadá.

Valero, M. V., Prado, R. M., Zawadski, F., Eiras, C. E., Madrona, G. S. \& Prado, I. N. (2014). Propolis and essential oils additives in the diets improved animal performance and feed efficiency of bulls finished in feedlot. Acta Scientiarum Animal Sciences, 32, 419-426.

Volp, A. C. P., Renhe, I. R. T. \& Stringueta, P. C. (2011). Carotenoides: pigmentos naturais como compostos bioativos. Revista Brasileira de Nutrição Clinica, 26(4), 291-298.

Wahlin, S., Floderus, Y., Stål, P. \& Harper, P. (2011). Erythropoietic protoporphyria in Sweden: demographic, clinical, biochemical and genetic characteristics. Journal of Internal Medicine, 269(3), 278-288.

Xie, Y., Li, W., Zhu, L., Zhai, S., Qin, S. \& Du, Z. (2019). Effects of phycocyanin in modulating the intestinal microbiota of mice. Microbiology, 8(9), 1-11.

Yang, C., Chowdhury, M.A., Huo, Y. \& Gong, J. (2015). Phytogenic Compounds as Alternatives to In-Feed Antibiotics: Potentials and Challenges in Application. Pathogens, 4(1), 137-156.

Yitbarek, M. B. (2015). Phytogenics as feed additives in poultry production: a review. International Journal of Extensive Research, 3, 49-60.

Zhang, G. G., Yang, Z. B., Wang, Y. \& Yang, W. R. (2013). Effects of Astragalus membranaceus root processed to different particle sizes on growth performance, antioxidant status, and serum metabolites of broiler chickens. Poultry Science, 92, 178-183.

Zhang, W., Xiao, S., Samaraweera, H., Lee, E. J. \& Ahn, D. U. (2010). Improving functional value of meat products. Meat Science, 86, 15-31. 